\title{
The persistent prevalence and evolution of cross-family recombinant coronavirus GCCDC1 among a bat population: a two-year follow-up
}

\author{
Joseph O. Obameso ${ }^{1,3 \dagger}$, Hong $\mathrm{Li}^{2 \dagger}$, Hao Jia ${ }^{4,5}$, Min Han ${ }^{1}$, Shiyan Zhu ${ }^{4,5}$, Canping Huang ${ }^{5}$, \\ Yuhui Zhao ${ }^{1}$, Min Zhao ${ }^{1,3}$, Yu Bai ${ }^{1,3}$, Fei Yuan ${ }^{1}$, Honglan Zhao ${ }^{5}$, Xia Peng ${ }^{2}$, Wen Xu ${ }^{2}$, \\ Wenjie Tan $^{5}$, Yingze Zhao ${ }^{5}$, Kwok-Yung Yuen ${ }^{6}$, William J. Liu, ${ }^{4,5}$, Lin Lu $^{2 *}$ \& George F. Gao ${ }^{1,3,5^{*}}$ \\ ${ }^{1}$ CAS Key Laboratory of Pathogenic Microbiology and Immunology, Institute of Microbiology, Chinese Academy of Sciences, \\ Beijing 100101, China; \\ ${ }^{2} Y u n n a n$ Provincial Center for Disease Control and Prevention, Kunming 650022, China; \\ ${ }^{3}$ University of Chinese Academy of Sciences, Beijing 100049, China; \\ ${ }^{4}$ College of Laboratory Medicine and Life Sciences, Wenzhou Medical University, Wenzhou 325035, China; \\ ${ }^{5}$ Key Laboratory of Medical Virology and Viral Diseases, Ministry of Health of People's Republic of China, National Institute for Viral Disease \\ Control and Prevention, Chinese Center for Disease Control and Prevention, Beijing 102206, China; \\ ${ }^{6}$ State Key Laboratory of Emerging Infectious Diseases, The University of Hong Kong, Hong Kong Special Administrative Region, \\ Hong Kong, China
}

Received September 11, 2017; accepted October 23, 2017; published online December 1, 2017

\begin{abstract}
Bats are connected with the increasing numbers of emerging and re-emerging viruses that may break the species barrier and spread into the human population. Coronaviruses are one of the most common viruses discovered in bats, which were considered as the natural source of recent human-susceptible coronaviruses, i.e. SARS-COV and MERS-CoV. Our previous study reported the discovery of a bat-derived putative cross-family recombinant coronavirus with a reovirus gene $p 10$, named as Ro-BatCoV GCCDC1. In this report, through a two-year follow-up of a special bat population in one specific cave of south China, we illustrate that Ro-BatCoV GCCDC1 persistently circulates among bats. Notably, through the longitudinal observation, we identified the dynamic evolution of Ro-BatCoV GCCDC1 in bats represented by continuously recombination events. Our study provides the first glimpse of the virus evolution in one longitudinally observed bat population cohort and underlines the surveillance and pre-warning of potential interspecies transmittable viruses in bats.
\end{abstract}

coronavirus, bat population, evolution

Citation: $\quad$ Obameso, J.O., Li, H., Jia, H., Han, M., Zhu, S., Huang, C., Zhao, Y., Zhao, M., Bai, Y., Yuan, F., Zhao, H., Peng, X., Xu, W., Tan, W., Zhao, Y., Yuen, K. Y., Liu, W. J., Lu, L., and Gao, G.F. (2017). The persistent prevalence and evolution of cross-family recombinant coronavirus GCCDC1 among a bat population: a two-year follow-up. Sci China Life Sci 60, 1357-1363. https://doi.org/10.1007/s11427-017-9263-6

\section{INTRODUCTION}

Bats (Order Chiroptera) are thepg only group of mammals

$\dagger$ Contributed equally to this work

*Corresponding authors (Lin Lu, email: lulin@yncdc.cn; George F. Gao, email: gaofu@chinacdc.cn) capable of flight with the ability to circumnavigate at night, $i$. e., nocturnal animals (Calisher et al., 2006). Bats have been the focus of particular interest not just because of their ecological interest, but also because of their medical importance in public health, as they play significant role in evolving and transmission of zoonotic viral agents that 
stance a substantial danger to human and animal well-beings. Bats possess special peculiarities and features which make them host to different viruses, such features including structural diversity and complex lifestyle, diverse mode of feeding ranging from insectivores (feed on insects), frugivore (fruit eater), nectarivore (sucking nectar), palynivore (eating pollen), piscivore (eating fish) while others feed on arthropods, vertebrates or practice haematophagy (feed on blood). Bats are social animals that live in a densely populated roost, demographic and spatial structure of population with long life, which may significantly contribute to maintaining viruses as well as the high possibility of intra- and inter-species transmission of viral infections (Calisher et al., 2006). In addition, excreta during migration may be an important factor in the spread of viruses (López-Roig et al., 2014).

Bats are connected with the increasing numbers of emerging and re-emerging viruses including Hendra virus $(\mathrm{HeV})$, Nipah virus (NiV) and Ebola virus (EBOV), and also coronaviruses, etc. (Li et al., 2016). The studies of bats as reservoirs for a number of mammalian coronaviruses came to the spotlight since the discovery of severe acute respiratory syndrome (SARS)-like coronaviruses in 2005 isolated from south China (Lau et al., 2005; Li et al., 2005), and subsequently, a SARS-CoV receptor ACE2-using coronavirus was isolated from bat (Ge et al., 2013). Sister to SARS-CoV was the middle east respiratory syndrome-associated coronavirus (MERS-CoV) emerged in 2012, the close-related viruses of which was also found in bats (Lelli et al., 2013; Zumla et al., 2016). Actually, a number of bat coronaviruses have been documented in recent years (Hu et al., 2017), and the MERS$\mathrm{CoV}$ phylogenetically closely related bat coronaviruses (BatCoVs) HKU4 can also recognize human CD26 for cell entry, indicating origin of human-susceptible coronaviruses from bats (Wang et al., 2014).

Coronavirus encrypts a non-segmented, largest positive sense single-stranded RNA genome known ranging from 27 to $32 \mathrm{~kb}$ in length (Lai, 1996; Sokolova et al., 1996; Du et al., 2016). In the subfamily of Coronaviridae, Alphacoronavirus and Betacoronavirus infect and cause diseases in mammals, while Gammacoronavirus and Deltacoroanvirus are mostly confined to avian infections and diseases (Jackwood et al., 2012; Woo et al., 2012). Report has shown that coronaviruses possess low proofreading capability that can also exhibit comparatively high mutation and recombinant rates, which make them one of the most diverse, genetically distinct, and recently emerging groups of viruses (Minskaia et al., 2006). The continuous mutation and recombination of the coronaviruses probably bridge the species barrier and enable them to spread into the human population (Baric et al., 1995).

Recently, we discovered a novel Rousettus bat coronavirus GCCDC1 (Ro-BatCoV GCCDC1) with full genome sequenced, which exhibits a putative heterologous re- combination event between a bat coronavirus and a bat orthoreovirus (Huang et al., 2016). We found that the p10 gene of bat orthoreovirus in Ro-BatCoV GCCDC1 genome has no homologous gene from any parental strain of coronaviruses. However, there is dearth of information whether this virus with the recombinant gene can persist and evolve in their hosts.

Herein we carried out a longitudinal survey on one population of Roussetus leschenaulti bats in Yunnan Province, Mainland China, between 2014 and 2015. The persistence of the Ro-BatCoV GCCDC1 in the bat population and its potential evolution was investigated. Our study provides the first glimpse of the virus evolution in one prospectively and longitudinally observed bat population.

\section{RESULTS}

\section{The persistent prevalence of the Ro-BatCoV GCCDC1 in the bats}

A total of 568 rectal swab samples from $R$. leschenaulti bats were collected between May $2014(n=118)$, October 2014 $(n=270)$ and October $2015(n=180)$ in one cave located in Xishuangbanna, Yunnan Province, China (Table 1). The anal swab samples were screened individually for the presence of coronavirus using RT-PCR detection method. Coronavirus positive ratios were $39.8 \%(47 / 118), 38.8 \%(70 / 270)$ and $35.6 \%(64 / 180)$ in bats sampled in May 2014, October 2014 and October 2015, respectively (Table 1). These results showed a persistent prevalence of Ro-BatCoV GCCDC1 in this bat population.

\section{The evolution of $R d R p$ from Ro-Bat GCCDC1}

Based on the identified PCR CoV-positive bat samples, the PCR amplicons with the length of 228 bp of Ro-BatCoV GCCDC1 $R d R p$ were sequenced, which showed the conservation of this newly identified bat $\mathrm{CoV}$. We analyzed the $R d R p$ sequences which had the corresponding $p 10$ sequences available, thus we have 97 pairs of $R d R p$ and $p 10$ from 97 bat specimens. The phylogenetic analysis of the $R d R p$ sequences revealed two groups of Ro-BatCoV GCCDC1 designated Group I (RdRp-I) and Group II (RdRp-II) (Figure 1A). The result from our study revealed that all the Ro-BatCoV GCCDC1 $R d R p$ from the first round sampling in May 2014 belonged to the RdRp-I, while subsequent sampling in October 2014 and 2015, respectively, showed the involvement of viruses featured with RdRp-II.

\section{The evolution of $p 10$ gene in Ro-BatCoV GCCDC1}

Based on the result of PCR amplification using specific primers, the nucleic acid sequences of $p 10$ genes ( $420 \mathrm{bp}$ ) of 
Table 1 Summary of sampling detail and coronavirus infections in bats ${ }^{\mathrm{a})}$

\begin{tabular}{ccc}
\hline Sampling time & $\begin{array}{c}\text { Bats with swab } \\
\text { samples collected }\end{array}$ & $\begin{array}{c}\text { Bats with CoV } \\
\text { positive (\%) }\end{array}$ \\
\hline May 2014 & 118 & $47(39.8 \%)$ \\
October 2014 & 270 & $70(38.8 \%)$ \\
October 2015 & 180 & $64(35.6 \%)$ \\
\hline
\end{tabular}

a) *, All the positive samples were defined based on the PCR amplicons with the length of 228 bp of Ro-BatCoV GCCDC1 $R d R p$ as described in Table 2. $97 R d R p$ sequences out of the 181 samples which had the corresponding $p 10$ sequences were utilized for the GCCDC evolution.

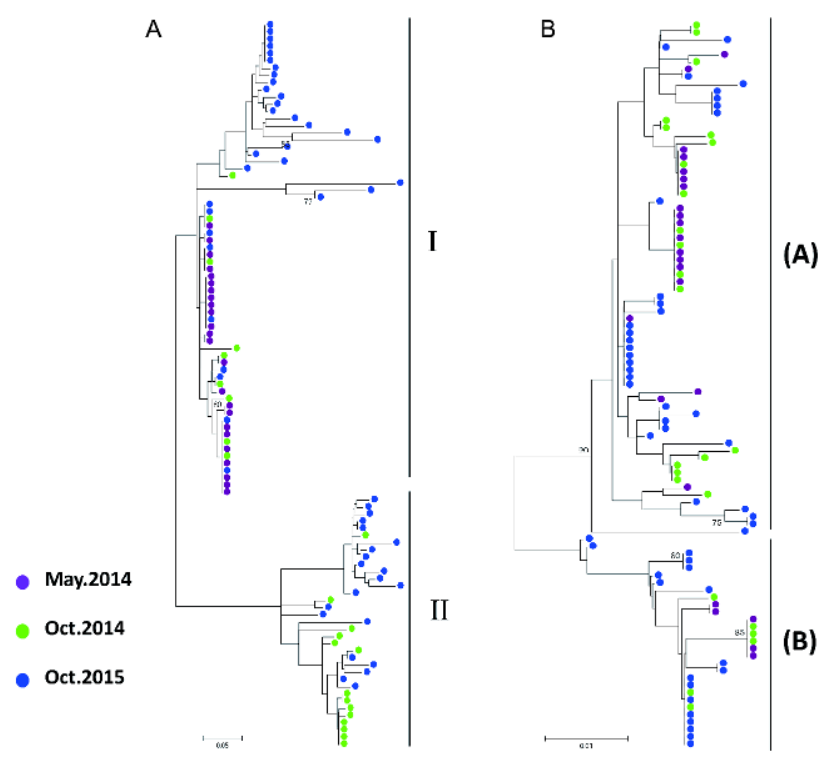

Figure 1 Phylogenetic analyses of Ro-BatCoV GCCDC1 for three sampling periods. Evolutionary trees of $R d R p(\mathrm{~A})$ and $P 10(\mathrm{~B})$ constructed for the samples collected from May, 2014 to Oct., 2015 has indicated by different colors as labeled. The maximum-likelihood trees were constructed with 1,000 replication available in MGEA6.

Ro-BatCoV GCCDC1 were subjected to phylogenetic analysis, which displayed high level of sequence similarity to Ro-BatCoV GCCDC1 p10 genome segments (Huang et al., 2016). In pairwise comparison of nucleotide sequence, the nucleotide sequence identity was $99 \%$ compared to RoBatCoV GCCDC1. However, based on the phylogenetic analysis of all the available Ro-BatCoV GCCDC1 in public domain, the 10 genes were divided into two groups: Group (A) and Group (B) (Figure 1B). All the three times of sampling Ro-BatCoV GCCDC1 covered both Group A and Group B, which indicated the persistence of the groups of p10 in Ro-BatCoV GCCDC1.

When compared to $p 10$ genes from traditional orthoreoviruses (Figure 2), the $p 10$ from the typical six Ro-BatCoV GCCDC1: GCCDC1/2-53 and GCCDC1/2-110 sampled in October 2014, and GCCDC1/3-19 and GCCDC1/3-31 in October 2015 and our previously identified Ro-BatCoV GCCDC1 strains 346-KU762337 and Ro-BatCoV GCCDC1 strains 356-KU762338, are clustered into one independent branch indicating the uniqueness of the bat coronavirus-derived $p 10$ gene. However, we can still observe that $p 10$ genes in 2-53 and 3-31 belong to Group A and p10 of other four samples are in Group B.

\section{The potential dynamic recombination of Ro-BatCoV GCCDC1}

To further investigate the dynamic evolution of Ro-BatCoV GCCDC1 in the longitudinally collected bat samples, we further analyzed the potential recombination of the viruses by constructing a model that pairs of $R d R p$ and $p 10$ genes, each pair of which is sequenced from single Ro-BatCoV GCCDC1 sample. Five different nonsynonymous mutations were found in all available RdRp protein segments (Figure $3 \mathrm{~A})$. However, only two mutations were featured by the two groups of RdRp (T-648 and I-666 for RdRp-I and A-648 and M-666 for RdRp-II). Comparison of the translated amino acid sequences of $p 10$ gene of both Group A and B of RoBatCoV GCCDC1 identified four nonsynonymous mutations at position 7, 14, 19 and 34, respectively (Figure 3B). Group A p10 was featured with the combination of M-7, T14, A-19, and I-34, while Group B possesses substitutions V7, T-14, N-19 and S-34 in the four positions. When we paired the corresponding $R d R p$ and $p 10$ genes groups with their featured substitutions, there were four different combinations of the putative assembled genomes in all the bat samples tested (Figure 4A). Ro-BatCoV GCCDC1 virus IA possesses Group I of RdRp gene and Group A of $p 10$ gene. Ro-BatCoV GCCDC1 virus IB is featured with Group I of $R d R p$ gene and Group B of $p 10$ gene. Ro-BatCoV GCCDC1 virus IIA has Group II of $R d R p$ gene and Group A of $p 10$ gene. Ro-BatCoV GCCDC1 virus IIB characterizes by Group II of RdRp gene and Group B of $p 10$ gene. During the first round of sampling in May 2014, only viruses IA and IB were discovered in the bat population (Figure 4B). However, five months later in October 2014 IIA and IIB recombinants can be found in the bat swabs. Further, the third round sampling in October 2015 showed that while the four combinations were persistent in the bat population, the two viruses IIA and IIB became more dominant.

\section{DISCUSSION}

In our previous study, we demonstrated that a novel coronavirus Ro-BatCoV GCCDC1 encoded a putatively recombinant nonstructural protein $\mathrm{p} 10$ derived from reovirus, which induces the formation of syncytia in infected cells (Huang et al., 2016). Sequel to this, we now report that RoBatCoV GCCDC1 is continuously circulating in bats from Yunnan Province in China. We observed high prevalence of the Ro-BatCoV GCCDC1 on a consistent trend for a period 


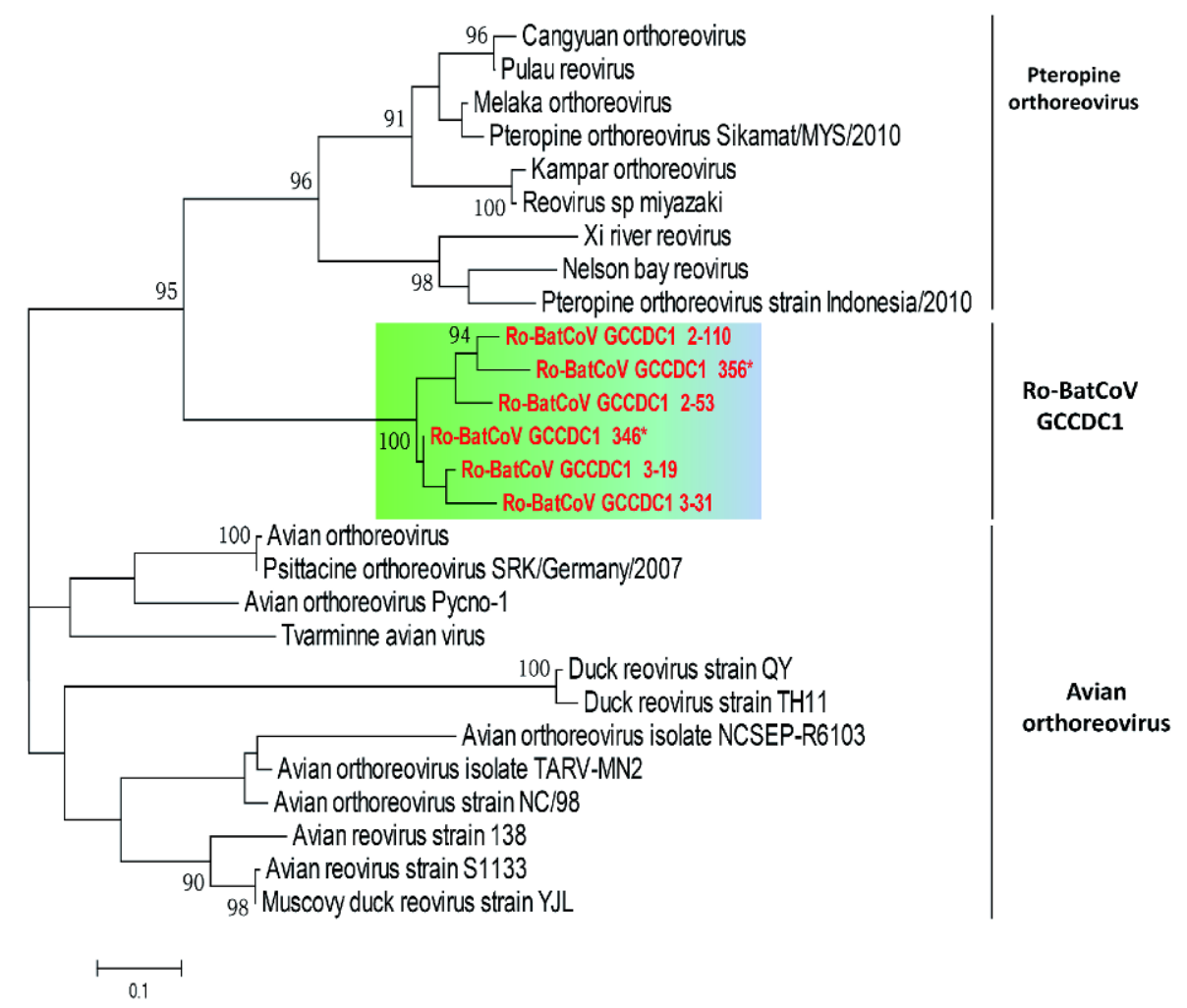

Figure 2 Phylogenetic relationships of Ro-BatCoV GCCDC1 compared to other viruses. Phylogenetic trees of the p10 sequence of Ro-BatCoV GCCDC1 together with the $p 10$ genes from other reoviruses. The phylograms were constructed by using MEGA6 software package to determine the maximumlikelihood tree for the sequence.
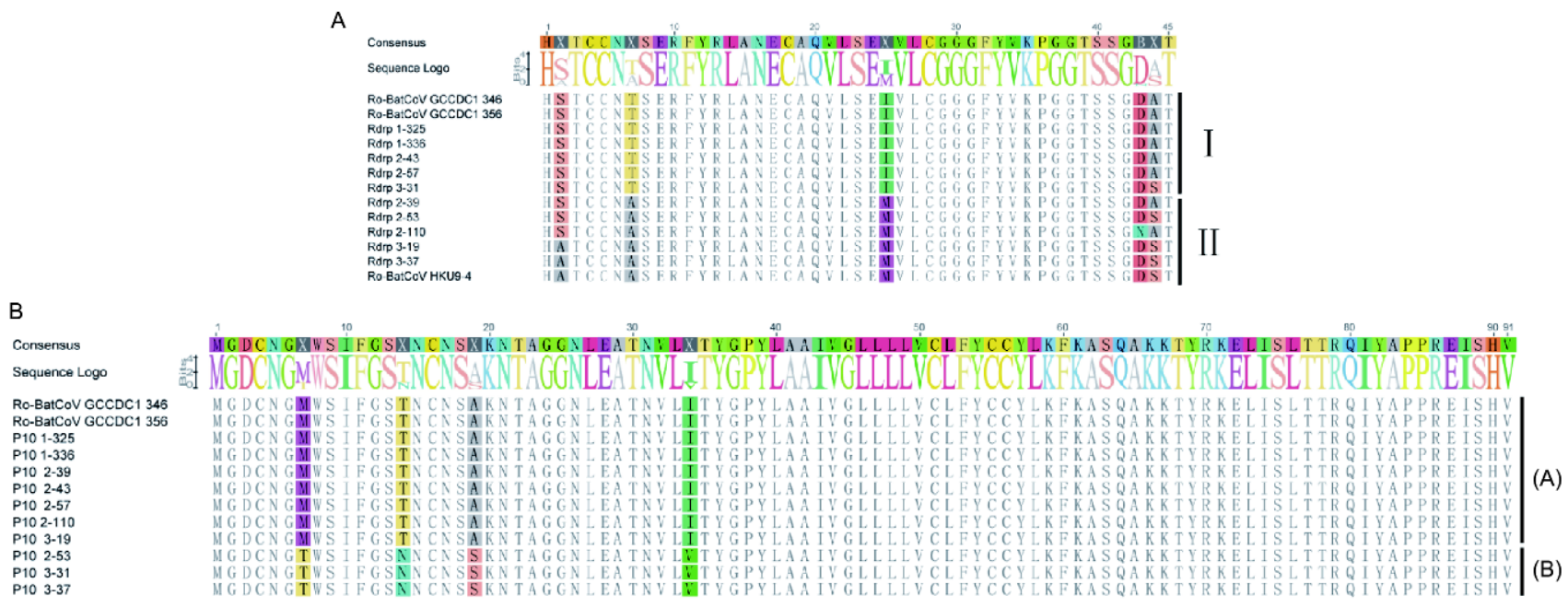

Figure 3 Multiple sequence alignment of Ro-BatCoV GCCDC1 genes based on samples from three sampling periods. Sequence alignment of deduced amino acid of RdRp (A) and p10 proteins (B). Consensus and sequence logo for conserved amino acid residues are shown on top of the alignment. The residues with color indicate the position of mutation.

of 2 years that is relatively high in proportion to the populations under study. The cross-family recombinant $p 10$ gene stably exists in the novel virus. Notably, we observed the dynamic evolution of this virus in the special bat population. Our study provides beneficial recommendation for the surveillance and pre-warning of potential interspecies transmittable viruses in bats.
The recombination events in coronavirus involving interchange of intra-family genetic materials has been reported (Baric et al., 1995). In our previous study, a traditional reovirus gene $p 10$ located at the upstream of $\mathrm{N}$ protein and downstream of NS7a of Ro-BatCoV GCCDC1 genome showing the ability of coronavirus to undergo a heterologous inter-family recombination (Huang et al., 2016). In our 


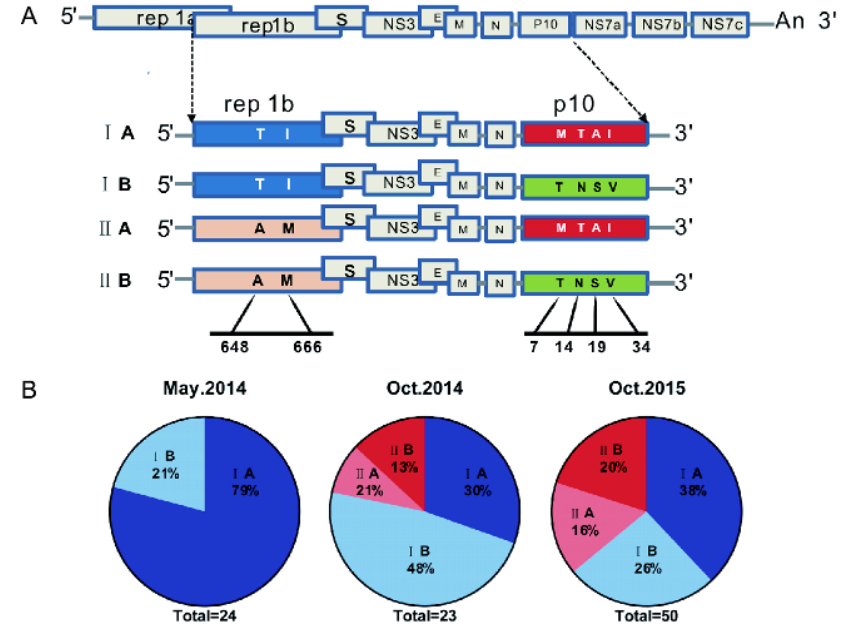

Figure 4 Recombination analysis of Ro-BatCoV GCCDC1. A, The recombination model for Ro-BatCoV GCCDC1 based on the different groups of RdRp (Group I and Group II) and p10 proteins (Group A and Group B). The groups are based on the different combinations of polymorphic amino acid residues in Ro-BatCoV GCCDC1. B, The ratios of different recombinants of Ro-BatCoV GCCDC1 revealed two new groups, IIA and IIB

current study, we observed a persistent prevalence Ro-Bat$\mathrm{CoV}$ GCCDC1 in the bat population, and we also found that the recombinant $p 10$ is a constitutive gene in this virus. We did not get all the $p 10$ sequences from the $R d R p$ positive samples. This may be due to a lower amplification efficiency of the 10 primers. Thus, the deep sequencing of the whole genomes and also the virus isolation from the original samples are still needed to elucidate more features of Ro-BatCoV GCCDC1.

The emergence of recombinant $p 10$ gene in Ro-BatCoV GCCDC1 could be due to unhindered access of reoviruses in bat, being a prehistoric mammal which might have favoured viral integration and persistence. p10 persistence may have utilized intrinsic factors mimicking ability to incorporate its genome into coronavirus or of the host's cells. $p 10$ has been reported to share similarities with viroporins, which has the ability to form hydrophilic channels that allow low molecular weight hydrophilic molecules to cross the membrane thereby interrupt membrane integrity, breakdown ionic gradients and eventual release of essential compounds from the cell (Carrasco et al., 1989; Carrasco et al., 1993). This could lead to series of events that favor viral replication, encourage viral transmission and persistence of Ro-BatCoV GCCDC1 in bat population. Interestingly, the phylogenetic analysis revealed that two distinct groups of $\mathrm{p} 10$ with featured amino acids persistently exist in Ro-BatCov GCCDC1. However, the comparison of the two p10 in the pathogenesis of bat corornavirus need further investigations.

Summarily, the new Ro-BatCoV GCCDC1 virus is not only persistently prevalent in the bat population, but also exhibits continuous evolution. Probability also exists that Ro-BatCoV GCCDC1 might be under diverse selective pressures. The evolving bat coronaviruses could have seized the advantage of conserved mammalian receptors for cellular replication and other biological pathways that go through radiation over a long time which could boost the capability for conveyance of bat-related viruses to other animals (Calisher et al., 2006). Our study benefits the understanding of dynamic virus evolution in special bat population and underlines the surveillance of potential interspecies viruses in bats.

\section{MATERIALS AND METHODS}

\section{Sample collection, storage and transportation}

Anal swab samples were collected from Rousettus leschenaulti ( $R$. leschenaulti) bats captured on three occasions, May 2014, October 2014 and October 2015, in one cave located in Xishuangbanna, Yunnan Province, China. Rectal swabs were collected from each bat and placed in viral transport medium (VTM) in screw cap tubes, kept on cold ice (at $4^{\circ} \mathrm{C}$ to $10^{\circ} \mathrm{C}$ ) and transported to the laboratory at local CDC where they were frozen in liquid nitrogen. The VTM containing Earle's Balanced Salt Solution, 5\% bovine albu$\min , 50,000 \mu \mathrm{g} \mathrm{mL}^{-1}$ vancomycin, $50,000 \mu \mathrm{g} \mathrm{mL}^{-1}$ amikacin, 10,000 units $\mathrm{mL}^{-1}$ nystatin as described in our previous study (Huang et al., 2016). The samples were later transported to the laboratory in dry ice and stored at $-80^{\circ} \mathrm{C}$ until further analysis.

\section{RNA extraction}

VTM containing swab samples were manipulated by centrifugation at $8,000 \mathrm{r} \mathrm{min}^{-1}$ for $5 \mathrm{~min}$ at $4{ }^{\circ} \mathrm{C}$ using a Thermal centrifuge. Total RNA was extracted from $140 \mu \mathrm{L}$ of the supernatants using the RNeasy mini kit (Qiagen, Germany) according to the manufacturer's protocol. The RNA was eluted in $60 \mu \mathrm{L}$ AVE buffer and stored at $-80^{\circ} \mathrm{C}$ for subsequent use.

\section{Viral detection by nested-PCR}

The extracted total RNA was transcribed to cDNA using reverse transcriptase enzyme (Thermo, USA) and then screened for the presence of coronavirus RNA using pancoronavirus RT-PCR degenerate primers. The primers were designed from a highly conserved RdRp region of coronaviruses (Table 2). Theoretically, this pan-coronavirus RTPCR primers should amplify a $299 \mathrm{bp}$ fragment of the polymerase gene of all coronaviruses in the first round and $228 \mathrm{bp}$ in a semi-nested PCR for the second round. If the samples were RT-PCR positive to coronavirus screening, specific p10 primers (Table 2) that covered the whole $p 10$ gene were used to screen for the presence of reovirus $p 10$ 
Table 2 The primers for coronaviridae gene identification and amplification

\begin{tabular}{|c|c|c|c|c|c|}
\hline Primer name & $\begin{array}{l}\text { Target } \\
\text { gene }\end{array}$ & Sequence $\left(5^{\prime} \rightarrow 3^{\prime}\right)$ & $\mathrm{G}+\mathrm{C}(\%)$ & $\mathrm{T}_{\mathrm{m}}\left({ }^{\circ} \mathrm{C}\right)$ & $\begin{array}{c}\text { Length of the amplification } \\
\text { fragments }\end{array}$ \\
\hline$R d R p-\mathrm{OF}$ & $R d R p$ & TGTTATTGGAACCACGAAGTTYTAYGGNGGNTG & 42.1 & 51.3 & $\mathrm{OF}+\mathrm{OR}=299 \mathrm{bp}$ \\
\hline$R d R p$-IF & $R d R p$ & GTTTTGTTCTTATGGGTTGGGATTAYCCNAARTGYGA & 42.1 & 52.3 & $\mathrm{IF}+\mathrm{OR}=228 \mathrm{bp}$ \\
\hline$R d R p-\mathrm{OR}$ & $R d R p$ & TAGTAGCATCTCCGCTGCTAGTNCCNCCNGGYTT & 52.6 & 53.4 & \\
\hline $\mathrm{CoV}-\mathrm{p} 10-\mathrm{OF}$ & p10 & GAAAGGGAGACTAAGAAGA & 42.1 & 50.8 & \multirow{2}{*}{$\mathrm{OF}+\mathrm{OR}=715 \mathrm{bp}$} \\
\hline CoV-p10-OR & p10 & ACAGGCTATAACTGTAAATG & 35.0 & 49.2 & \\
\hline CoV-p10-IF & p10 & GCAGATGAGAATGACAAACCCAA & 43.5 & 56.0 & \multirow{2}{*}{$\mathrm{IF}+\mathrm{IR}=447 \mathrm{bp}$} \\
\hline CoV-p10-IR & p10 & GCAGCAAGCAAGCAGAGCAA & 55.0 & 57.4 & \\
\hline
\end{tabular}

gene in Ro-BatCoV GCCDC1. The amplified PCR products were purified following electrophoresis in $1 \%$ agarose gels that revealed the expected amplicon in agarose gels.

\section{Virus isolation}

RT-PCR positive samples for coronavirus were cultured in human epithelial colorectal adenocarcinoma cells ( $\mathrm{CaCo}-2)$, human lung carcinoma cells (A549), human epithelial type 2 HeLa derivative cells (HEp-2), baby hamster kidney (BHK21) and rhesus monkey kidney cells (LLC-mk2). Cells were grown in Dulbecco's modified Eagle's minimum essential medium (DMEM) supplemented with $10 \%$ or $20 \%$ fetal bovine serum (FBS) and incubated at $37^{\circ} \mathrm{C}$ with $5 \% \mathrm{CO}_{2}$. The cell lines were inoculated with the supernatant of PCR positive samples in 24-well plates, incubated for $2 \mathrm{~h}$ for virus adsorption, $300 \mu \mathrm{L}$ fresh cell culture medium with $2 \%$ FBS was added and incubated at $37^{\circ} \mathrm{C}$ in $5 \% \mathrm{CO}_{2}$ incubator. The plates were observed daily for viral cytopathic effect (CPE).

\section{Molecular and sequencing analysis}

The used reference sequences were downloaded from GenBank. Multiple sequence alignment of nucleic acid sequences was performed using ClustalW and the Geneious program (http://www.geneious.com). For $R d R p$ and $p 10$ phylogenetic analysis, maximum-likelihood trees were constructed with MEGA 6 (http://www.megasoftware.net), and 1,000 bootstrap replicates were run.

Compliance and ethics The author(s) declare that they have no conflict of interest.

\footnotetext{
Acknowledgements We appreciate the great efforts of Drs. Yongming Zhou, Honghua Wen, Huaxing Liu, who participated in the collection of the bat samples. This work was supported by the National Key Research and Development Program of China (2017YFC1200202), the National Natural Science Foundation of China (81290342, 81461168030), the Major Special Projects for Infectious Disease Research of China (2016ZX10004222-003), and China National Grand S\&T Special Project (2014ZX10004-001-006). Joseph O. Obameso was supported by CAS-TWAS President's Fellowship of
}

the University of Chinese Academy of Sciences (UCAS) and The World Academy of Sciences (TWAS). George F. Gao is a leading principle investigator of the NSFC Innovative Research Group (81621091). The funders had no role in study design, data collection and analysis, decision to publish, or preparation of the manuscript.

Baric, R.S., Fu, K., Chen, W., and Yount, B. (1995). High recombination and mutation rates in mouse hepatitis virus suggest that coronaviruses may be potentially important emerging viruses. Adv Exp Med Biol 380, 571-576.

Calisher, C.H., Childs, J.E., Field, H.E., Holmes, K.V., and Schountz, T. (2006). Bats: important reservoir hosts of emerging viruses. Clin Microbiol Rev 19, 531-545.

Carrasco, L., Otero, M.J., and Castrillo, J.L. (1989). Modification of membrane permeability by animal viruses. Pharmacol Ther 40, 171-212.

Carrasco, L., Perez, L., Irurzun, A., Lama, J., Martinez-Abarca, F., Rodriguez, P., Guinea, R., Castrillo, J.L., Sanz, M.A., and Ayala, M.J. (1993). Regulation of Gene Expression in Animal Viruses. (New York: Plenum Publishing Corp.), pp. 283-305.

Du, J., Yang, L., Ren, X., Zhang, J., Dong, J., Sun, L., Zhu, Y., Yang, F., Zhang, S., Wu, Z., and Jin, Q. (2016). Genetic diversity of coronaviruses in Miniopterus fuliginosus bats. Sci China Life Sci 59, 604-614.

Ge, X.Y., Li, J.L., Yang, X.L., Chmura, A.A., Zhu, G., Epstein, J.H., Mazet, J.K., Hu, B., Zhang, W., Peng, C., Zhang, Y.J., Luo, C.M., Tan, B., Wang, N., Zhu, Y., Crameri, G., Zhang, S.Y., Wang, L.F., Daszak, P., and Shi, Z.L. (2013). Isolation and characterization of a bat SARS-like coronavirus that uses the ACE2 receptor. Nature 503, 535-538.

Hu, B., Zeng, L.P., Yang, X.L., Ge, X.Y., Zhang, W., Li, B., Xie, J.Z., Shen, X.R., Zhang, Y.Z., Wang, N., Luo, D.S., Zheng, X.S., Wang, M.N., Daszak, P., Wang, L.F., Cui, J., and Shi, Z.L. (2017). Discovery of a rich gene pool of bat SARS-related coronaviruses provides new insights into the origin of SARS coronavirus. PLoS Pathog 13, e1006698.

Huang, C., Liu, W.J., Xu, W., Jin, T., Zhao, Y., Song, J., Shi, Y., Ji, W., Jia, H., Zhou, Y., Wen, H., Zhao, H., Liu, H., Li, H., Wang, Q., Wu, Y., Wang, L., Liu, D., Liu, G., Yu, H., Holmes, E.C., Lu, L., and Gao, G.F. (2016). A bat-derived putative cross-family recombinant coronavirus with a reovirus gene. PLoS Pathog 12, e1005883.

Jackwood, M.W., Hall, D., and Handel, A. (2012). Molecular evolution and emergence of avian gammacoronaviruses. Infect Genet Evol 12, 13051311.

Lai, M.M.C. (1996). Recombination in large RNA viruses: coronaviruses. Semin Virol 7, 381-388.

Lau, S.K.P., Woo, P.C.Y., Li, K.S.M., Huang, Y., Tsoi, H.W., Wong, B.H.L., Wong, S.S.Y., Leung, S.Y., Chan, K.H., and Yuen, K.Y. (2005). Severe acute respiratory syndrome coronavirus-like virus in Chinese horseshoe bats. Proc Natl Acad Sci USA 102, 14040-14045.

Lelli, D., Papetti, A., Sabelli, C., Rosti, E., Moreno, A., and Boniotti, M.B. (2013). Detection of coronaviruses in bats of various species in italy. Viruses 5, 2679-2689.

Li, W., Shi, Z., Yu, M., Ren, W., Smith, C., Epstein, J.H., Wang, H., 
Crameri, G., Hu, Z., Zhang, H., Zhang, J., McEachern, J., Field, H., Daszak, P., Eaton, B.T., Zhang, S., and Wang, L.F. (2005). Bats are natural reservoirs of SARS-like coronaviruses. Science 310, 676-679.

Li, Z., Liu, D., Ran, X., Liu, C., Guo, D., Hu, X., Tian, J., Zhang, X., Shao, Y., Liu, S., and Qu, L. (2016). Characterization and pathogenicity of a novel mammalian orthoreovirus from wild short-nosed fruit bats. Infect Genet Evol 43, 347-353.

López-Roig, M., Bourhy, H., Lavenir, R., and Serra-Cobo, J. (2014). Seroprevalence dynamics of european bat lyssavirus type 1 in a multispecies bat colony. Viruses 6, 3386-3399.

Minskaia, E., Hertzig, T., Gorbalenya, A.E., Campanacci, V., Cambillau, C., Canard, B., and Ziebuhr, J. (2006). Discovery of an RNA virus $3^{\prime} \rightarrow 5^{\prime}$ exoribonuclease that is critically involved in coronavirus RNA synthesis. Proc Natl Acad Sci USA 103, 5108-5113.

Sokolova, T.M., Uryvaev, L.V., Selivanova, T.K., Bobrova, O.V., Lebedev, A., and Bystrov, N.S. (1996). Recombination of alpha-viruses during mixed infection in cultured cells. Formation of hybrid forms of RNA of
Venezuelan equine encephalomyelitis virus and Karelian fever virus in the region of genes for nucleocapsid and envelope proteins. Vopr Virusol 41, 245-252.

Wang, Q., Qi, J., Yuan, Y., Xuan, Y., Han, P., Wan, Y., Ji, W., Li, Y., Wu, Y., Wang, J., Iwamoto, A., Woo, P.C., Yuen, K.Y., Yan, J., Lu, G., and Gao, G.F. (2014). Bat origins of MERS-CoV supported by bat coronavirus HKU4 usage of human receptor CD26. Cell Host Microbe 16, 328-337.

Woo, P.C.Y., Lau, S.K.P., Lam, C.S.F., Lau, C.C.Y., Tsang, A.K.L., Lau, J. H.N., Bai, R., Teng, J.L.L., Tsang, C.C.C., Wang, M., Zheng, B.J., Chan, K.H., and Yuen, K.Y. (2012). Discovery of seven novel mammalian and avian coronaviruses in the genus deltacoronavirus supports bat coronaviruses as the gene source of alphacoronavirus and betacoronavirus and avian coronaviruses as the gene source of gammacoronavirus and deltacoronavirus. J Virol 86, 3995-4008.

Zumla, A., Chan, J.F.W., Azhar, E.I., Hui, D.S.C., and Yuen, K.Y. (2016). Coronaviruses - drug discovery and therapeutic options. Nat Rev Drug Discov 15, 327-347. 